\title{
Conformational Studies by Dynamic NMR. 100. ${ }^{1}$ Enantiomerisation Process of Stereolabile Atropisomers in Pyridine-Substituted Adamantane Derivatives.
}

Daniele Casarini, ${ }^{\#}$ Carmine Coluccini, ${ }^{2, \dagger}$ Lodovico Lunazzi, ${ }^{\dagger}$ Andrea Mazzanti, ${ }^{* \dagger}$ and Romina Rompietti,

Chemistry Department, University of Basilicata, Potenza, Italy, Department of Organic Chemistry "A. Mangini”, University of Bologna, Viale Risorgimento 4, Bologna 40136, Italy and Dipartimento di Studi di Chimica e Tecnologia delle Sostanze Biologicamente Attive, Università "La Sapienza", P.le A. Moro 5, Roma 00185 Italy.

mazzand@ms.fci.unibo.it

Page S2 X-ray crystal data of compound $\mathbf{3}$

Page S3 Calculated and experimental torsional angles for ground state and transition state for compounds 1-6

Page S4 Table of rate constants for the simulated spectra of compound 1

Page S5-S10 MMX calculated ground state for compounds 1-6

Page S11-S16 MMX calculated transition state for compounds 1-6 


\section{Crystal Data for 2-Pyridin-3-yl-adamantan-2-ol (3)}

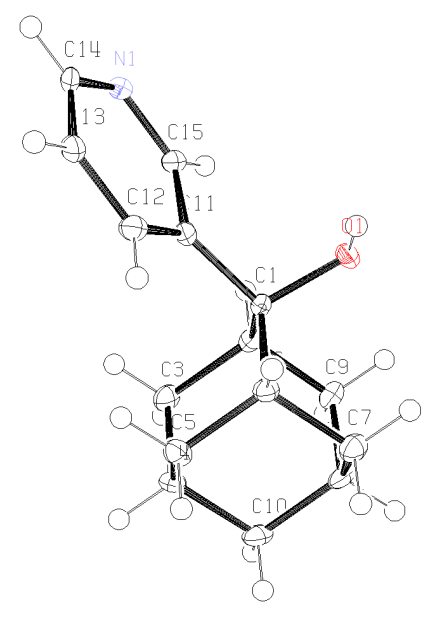

Molecular formula: $\mathrm{C}_{15} \mathrm{H}_{19} \mathrm{NO}, M_{\mathrm{r}}=229.31$, tetragonal, space group $P 4{ }_{1}{ }_{1} 2$ (No. 92), $a=$ 5.5986(7), $c=53.743(8) \AA, V=2340.0(5) \AA^{3}, \mathrm{~T}=100(2) \mathrm{K}, Z=8, \rho_{\mathrm{c}}=1.302 \mathrm{~g} \mathrm{~cm}^{-3}, F(000)=$ 992, graphite-monochromated $\mathrm{Mo}_{\mathrm{K} \alpha}$ radiation $(\lambda=0.71073 \AA), \mu\left(\mathrm{Mo}_{\mathrm{K} \alpha}\right)=0.081 \mathrm{~mm}^{-1}$, colourless plates $\left(0.4 \times 0.4 \times 0.15 \mathrm{~mm}^{3}\right)$, empirical absorption correction with SADABS (transmission factors: 0.992 - 0.9684), 1800 frames, exposure time $30 \mathrm{~s}, 1.52 \leq \theta \leq 30.0,-8 \leq h \leq 9,-9 \leq k \leq 9,-75 \leq l \leq$ 75,24285 reflections collected, 3342 independent reflections $\left(R_{\text {int }}=0.0471\right), 3318$ reflections with $I$ $>2 \sigma(I)\left(R_{\sigma}=0.0482\right)$, solution by direct methods (SHELXS97 $)$ and subsequent Fourier syntheses, full-matrix least-squares on $F_{\mathrm{o}}{ }^{2}\left(\mathrm{SHELX} 97^{\mathrm{a}}\right)$, hydrogen atoms refined with a riding model excepted for the hydroxyl group, data / parameters $=3342 / 158, S\left(F^{2}\right)=1.319, R(F)=0.0777$ and $w R\left(F^{2}\right)=$ 0.1718 on all data, $R(F)=0.0781$ and $w R\left(F^{2}\right)=0.1720$ for reflections with $I>2 \sigma(I)$, weighting scheme $w=1 /\left[\sigma^{2}\left(F_{\mathrm{o}}{ }^{2}\right)+(0.0077 P)^{2}+5.337 P\right]$ where $P=\left(F_{\mathrm{o}}{ }^{2}+2 F_{\mathrm{c}}{ }^{2}\right) / 3$, largest difference peak and hole 0.3458 and -0.366 e $\AA^{-3}$. Flack parameter: $0(3)$; test on the enantiomorphic $P 4_{3} 2{ }_{1} 2$ spatial group gives Flack parameter 1(3). Crystallographic data (excluding structure factors) for the structure reported in this paper have been deposited with the Cambridge Crystallographic Data Centre as supplementary publication no. CCDC-238969. Copies of the data can be obtained free of charge on application to CCDC, 12 Union Road, Cambridge CB21EZ, UK (fax: (+44) 1223-336033; e-mail: deposit@ccdc.cam.ac.uk).

a) Sheldrick, G. M. SHELX97; Universität Göttingen, Germany, 1997.

c) Flack, H. D. Acta Crystallogr., Sect. A 1983, 39, 876. 
Calculated and experimental torsional angles for compouds 1-6

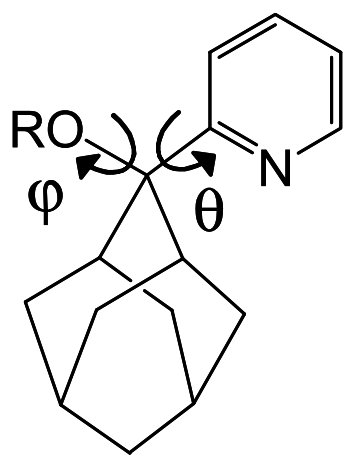

$1,2,4,6$

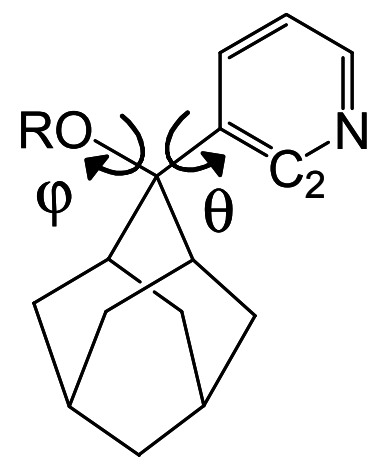

3,4

\begin{tabular}{|l|c|c|c|c|}
\hline Compound & \multicolumn{2}{|c|}{ Ground state } & \multicolumn{2}{c|}{ Transition state } \\
\hline & $\theta\left(^{\circ}\right)$ & $\varphi\left(^{\circ}\right)$ & $\theta$ & $\varphi$ \\
\hline $\mathbf{1}$ & 82 & -55 & 180 & -59 \\
\hline $\mathbf{2}$ & 105 & -59 & 180 & -59 \\
\hline $\mathbf{3}$ & 97 & -53 & 180 & -53 \\
\hline $\mathbf{3}-$ X ray & 63.5 & 55.4 & - & - \\
\hline $\mathbf{4}$ & 255 & -57 & 180 & -57 \\
\hline $\mathbf{5}$ & 107 & -61 & 180 & -61 \\
\hline $\mathbf{6}$ & 107 & -61 & 180 & -61 \\
\hline
\end{tabular}

$\theta=\mathrm{N}-\mathrm{Cq}-\mathrm{Cq}-\mathrm{O}$ for $\mathbf{1 , 2 , 4 , 6}$

$\theta=\mathrm{C} 2-\mathrm{Cq}-\mathrm{Cq}-\mathrm{O}$ for $\mathbf{3 , 4}$

$\varphi=\mathrm{R}-\mathrm{O}-\mathrm{Cq}-\mathrm{Cq}$ for $\mathbf{1 , 2 , 3 , 4 , 5 , 6}$ 
Table of rate constants for the simulated spectra of compound 1

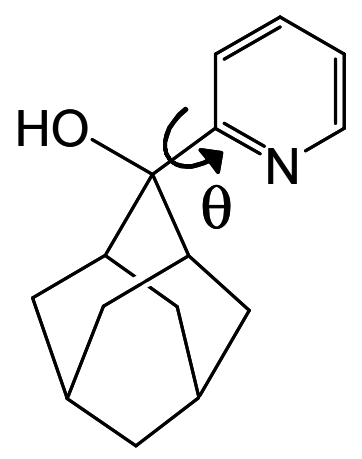

\begin{tabular}{|c|c|c|}
\hline Temperature $\left({ }^{\circ} \mathrm{C}\right)$ & Rate constant $\mathrm{s}^{-1}$ & Calculated $\Delta \mathrm{G}^{\mp}$ \\
\hline-30 & $\approx 10^{7}$ & $\approx 6.34$ \\
\hline-124 & 1250 & 6.40 \\
\hline-129 & 700 & 6.34 \\
\hline-136 & 250 & 6.30 \\
\hline-139 & 100 & 6.40 \\
\hline-143 & 55 & 6.36 \\
\hline-152 & $\leq 5$ & - \\
\hline
\end{tabular}




\section{2-Pyridin-2-yl-adamantan-2-ol (1):}

ground state

$\begin{array}{lrrr}\mathrm{C} & 0.113981 & -2.093026 & -1.224213 \\ \mathrm{C} & -1.198080 & -2.565154 & -1.872301 \\ \mathrm{C} & -2.295706 & -1.521530 & -1.607740 \\ \mathrm{C} & -2.492915 & -1.360502 & -0.093275 \\ \mathrm{C} & -1.181873 & -0.868877 & 0.551648 \\ \mathrm{C} & -0.091965 & -1.927487 & 0.291412 \\ \mathrm{C} & 0.527071 & -0.743910 & -1.836628 \\ \mathrm{C} & -1.875710 & -0.169090 & -2.203192 \\ \mathrm{C} & -0.727314 & 0.502611 & -0.021600 \\ \mathrm{C} & -0.562377 & 0.308126 & -1.550842 \\ \mathrm{C} & 1.476388 & 1.826236 & 0.065900 \\ \mathrm{C} & 0.507461 & 1.031153 & 0.700693 \\ \mathrm{~N} & 0.640732 & 0.794716 & 2.049626 \\ \mathrm{C} & 1.733576 & 1.226124 & 2.764185 \\ \mathrm{C} & 2.740229 & 1.952323 & 2.128537 \\ \mathrm{C} & 2.604683 & 2.262775 & 0.768724 \\ \mathrm{O} & -1.754514 & 1.455577 & 0.208837 \\ \mathrm{H} & -1.821079 & 1.555433 & 1.146315 \\ \mathrm{H} & 0.903008 & -2.837502 & -1.406397 \\ \mathrm{H} & -1.061916 & -2.722474 & -2.962735 \\ \mathrm{H} & -1.496864 & -3.557332 & -1.473990 \\ \mathrm{H} & -3.234719 & -1.855659 & -2.073151 \\ \mathrm{H} & -3.321239 & -0.651868 & 0.116621 \\ \mathrm{H} & -2.814973 & -2.321290 & 0.360049 \\ \mathrm{H} & -1.352214 & -0.738436 & 1.630521 \\ \mathrm{H} & -0.370985 & -2.897168 & 0.754235 \\ \mathrm{H} & 0.859698 & -1.637379 & 0.783866 \\ \mathrm{H} & 0.692885 & -0.847367 & -2.929394 \\ \mathrm{H} & 1.505023 & -0.414312 & -1.427588 \\ \mathrm{H} & -1.756124 & -0.251939 & -3.303725 \\ \mathrm{H} & -2.678754 & 0.583356 & -2.057306 \\ \mathrm{H} & -0.279261 & 1.280029 & -1.981251 \\ \mathrm{H} & 1.350628 & 2.109169 & -0.989625 \\ \mathrm{H} & 1.802048 & 0.991944 & 3.836787 \\ \mathrm{H} & 3.628235 & 2.275545 & 2.691539 \\ \mathrm{H} & 3.381698 & 2.847436 & 0.254506\end{array}$


ground state

$\begin{array}{lrrr}\mathrm{C} & 0.758602 & -2.495265 & -0.280335 \\ \mathrm{C} & -0.462506 & -3.401275 & -0.054112 \\ \mathrm{C} & -1.691847 & -2.525332 & 0.235542 \\ \mathrm{C} & -1.436689 & -1.665710 & 1.482575 \\ \mathrm{C} & -0.220216 & -0.745538 & 1.243715 \\ \mathrm{C} & 1.002779 & -1.646856 & 0.978115 \\ \mathrm{C} & 0.493857 & -1.565691 & -1.476288 \\ \mathrm{C} & -1.947640 & -1.596381 & -0.959242 \\ \mathrm{C} & -0.459494 & 0.241425 & 0.056500 \\ \mathrm{C} & -0.729023 & -0.675275 & -1.174655 \\ \mathrm{C} & 1.044724 & 1.743810 & -1.383976 \\ \mathrm{C} & 0.745338 & 1.159922 & -0.139977 \\ \mathrm{~N} & 1.547831 & 1.478300 & 0.931745 \\ \mathrm{C} & 2.653565 & 2.285964 & 0.806250 \\ \mathrm{C} & 2.981107 & 2.838529 & -0.430491 \\ \mathrm{C} & 2.161491 & 2.573213 & -1.535720 \\ \mathrm{O} & -1.662436 & 0.974535 & 0.351383 \\ \mathrm{C} & -1.548882 & 2.392758 & 0.424664 \\ \mathrm{H} & 1.657089 & -3.128932 & -0.486555 \\ \mathrm{H} & -0.643384 & -4.037127 & -0.953202 \\ \mathrm{H} & -0.275383 & -4.095747 & 0.798377 \\ \mathrm{H} & -2.583342 & -3.179498 & 0.402839 \\ \mathrm{H} & -2.348688 & -1.073741 & 1.732534 \\ \mathrm{H} & -1.252103 & -2.325682 & 2.363104 \\ \mathrm{H} & -0.055432 & -0.166867 & 2.186345 \\ \mathrm{H} & 1.186246 & -2.310625 & 1.855912 \\ \mathrm{H} & 1.933533 & -1.047939 & 0.850999 \\ \mathrm{H} & 0.313140 & -2.172363 & -2.395851 \\ \mathrm{H} & 1.400078 & -0.951447 & -1.687092 \\ \mathrm{H} & -2.137882 & -2.204746 & -1.875507 \\ \mathrm{H} & -2.875852 & -1.002970 & -0.788524 \\ \mathrm{H} & -0.961631 & -0.050842 & -2.068680 \\ \mathrm{H} & 0.404630 & 1.581500 & -2.263077 \\ \mathrm{H} & 3.278061 & 2.497592 & 1.690068 \\ \mathrm{H} & 3.866912 & 3.486129 & -0.536810 \\ \mathrm{H} & 2.389514 & 3.027111 & -2.514757 \\ \mathrm{H} & -1.242719 & 2.817934 & -0.560813 \\ \mathrm{H} & -0.849359 & 2.693263 & 1.242964 \\ \mathrm{H} & -2.556430 & 2.809345 & 0.671996\end{array}$


3-Pyridin-2-yl-adamantan-2-ol (3):

ground state

$\begin{array}{rrrr}\mathrm{C} & -1.293961 & 0.437859 & -0.404628 \\ \mathrm{C} & -2.159526 & 0.534316 & -1.676344 \\ \mathrm{C} & -1.506352 & -0.271134 & -2.808244 \\ \mathrm{C} & -0.101330 & 0.293042 & -3.067674 \\ \mathrm{C} & 0.748516 & 0.194802 & -1.784046 \\ \mathrm{C} & 0.123326 & 1.030736 & -0.635698 \\ \mathrm{C} & -1.196692 & -1.039966 & 0.016482 \\ \mathrm{C} & -1.406473 & -1.746992 & -2.389712 \\ \mathrm{C} & 0.851191 & -1.295037 & -1.398548 \\ \mathrm{C} & -0.551532 & -1.854904 & -1.116481 \\ \mathrm{H} & -1.838244 & 1.004151 & 0.386892 \\ \mathrm{H} & -3.185108 & 0.142210 & -1.476117 \\ \mathrm{H} & -2.288311 & 1.598042 & -1.986345 \\ \mathrm{H} & -2.124562 & -0.187386 & -3.736547 \\ \mathrm{H} & 0.385051 & -0.274003 & -3.896908 \\ \mathrm{H} & -0.170952 & 1.350930 & -3.414304 \\ \mathrm{H} & 1.760529 & 0.587980 & -2.042628 \\ \mathrm{H} & -2.211910 & -1.443739 & 0.243114 \\ \mathrm{H} & -0.599737 & -1.140704 & 0.953221 \\ \mathrm{H} & -2.424140 & -2.166573 & -2.207104 \\ \mathrm{H} & -0.952347 & -2.349715 & -3.211571 \\ \mathrm{H} & 1.492281 & -1.444874 & -0.500341 \\ \mathrm{H} & 1.335438 & -1.872654 & -2.221133 \\ \mathrm{H} & -0.474650 & -2.928015 & -0.810576 \\ \mathrm{~N} & 3.013192 & 0.778743 & 1.906919 \\ \mathrm{C} & 2.300838 & 0.756326 & 0.703651 \\ \mathrm{C} & 0.940426 & 1.106654 & 0.652365 \\ \mathrm{C} & 0.368537 & 1.626186 & 1.827981 \\ \mathrm{C} & 1.076121 & 1.659129 & 3.035890 \\ \mathrm{C} & 2.400551 & 1.216172 & 3.082758 \\ \mathrm{H} & 2.867709 & 0.449443 & -0.186193 \\ \mathrm{H} & -0.656381 & 2.027616 & 1.829944 \\ \mathrm{H} & 0.591796 & 2.038871 & 3.951579 \\ \mathrm{H} & 2.969251 & 1.219398 & 4.026729 \\ \mathrm{O} & -0.042022 & 2.493657 & -1.078362 \\ \mathrm{H} & 1.282353 & 3.196004 & -1.363975\end{array}$


ground state

\begin{tabular}{lrrr}
$\mathrm{O}$ & \multicolumn{1}{r}{} & & \\
$\mathrm{C}$ & 1.296725 & -0.272688 & -0.216771 \\
$\mathrm{C}$ & 2.192439 & -1.156677 & 0.675589 \\
$\mathrm{C}$ & 1.332646 & -2.204079 & 1.395680 \\
$\mathrm{C}$ & 0.269553 & -1.480914 & 2.235808 \\
$\mathrm{C}$ & -0.602640 & -0.591422 & 1.324661 \\
$\mathrm{C}$ & 0.234921 & 0.517573 & 0.615204 \\
$\mathrm{C}$ & 0.619099 & -1.172245 & -1.267231 \\
$\mathrm{C}$ & 0.649027 & -3.100747 & 0.350742 \\
$\mathrm{C}$ & -1.293379 & -1.518589 & 0.300672 \\
$\mathrm{C}$ & -0.237121 & -2.231679 & -0.556104 \\
$\mathrm{~N}$ & -2.662808 & 2.445830 & -0.991449 \\
$\mathrm{C}$ & -1.957439 & 1.632146 & -0.139786 \\
$\mathrm{C}$ & -0.569364 & 1.465087 & -0.279558 \\
$\mathrm{C}$ & 0.081276 & 2.281735 & -1.222300 \\
$\mathrm{C}$ & -0.647269 & 3.108159 & -2.087901 \\
$\mathrm{C}$ & -2.035728 & 3.171443 & -1.971856 \\
$\mathrm{O}$ & 0.972452 & 1.302191 & 1.565742 \\
$\mathrm{C}$ & 0.186807 & 2.159755 & 2.390241 \\
$\mathrm{H}$ & 1.988181 & 0.428593 & -0.740225 \\
$\mathrm{H}$ & 2.968133 & -1.667656 & 0.056769 \\
$\mathrm{H}$ & 2.752481 & -0.540925 & 1.417942 \\
$\mathrm{H}$ & 1.977617 & -2.831231 & 2.060232 \\
$\mathrm{H}$ & -0.365504 & -2.232096 & 2.763019 \\
$\mathrm{H}$ & 0.755402 & -0.878922 & 3.038907 \\
$\mathrm{H}$ & -1.389366 & -0.144360 & 1.975537 \\
$\mathrm{H}$ & -0.011837 & -0.566175 & -1.958760 \\
$\mathrm{H}$ & 1.388755 & -1.672105 & -1.902027 \\
$\mathrm{H}$ & 0.034244 & -3.882203 & 0.857128 \\
$\mathrm{H}$ & 1.414104 & -3.637969 & -0.258462 \\
$\mathrm{H}$ & -1.925581 & -2.271783 & 0.828185 \\
$\mathrm{H}$ & -1.985839 & -0.957213 & -0.366510 \\
$\mathrm{H}$ & -0.740634 & -2.880566 & -1.315433 \\
$\mathrm{H}$ & -2.515050 & 1.118091 & 0.656452 \\
$\mathrm{H}$ & 1.178817 & 2.299184 & -1.303553 \\
$\mathrm{H}$ & -0.128385 & 3.712526 & -2.850855 \\
$\mathrm{H}$ & -2.627800 & 3.809395 & -2.649338 \\
$\mathrm{H}$ & 0.875514 & 2.698551 & 3.084163 \\
$\mathrm{H}$ & -0.533292 & 1.578073 & 3.010117 \\
$\mathrm{H}$ & -0.347840 & 2.925581 & 1.781391 \\
& & &
\end{tabular}


ground state

$\begin{array}{lrrr}\mathrm{C} & 0.087250 & -2.276820 & -0.935989 \\ \mathrm{C} & -0.698431 & -3.333565 & -0.142368 \\ \mathrm{C} & -1.324138 & -2.671806 & 1.096258 \\ \mathrm{C} & -0.220144 & -2.062277 & 1.973236 \\ \mathrm{C} & 0.543790 & -0.983802 & 1.175628 \\ \mathrm{C} & 1.186636 & -1.685640 & -0.040180 \\ \mathrm{C} & -0.857470 & -1.148144 & -1.379975 \\ \mathrm{C} & -2.274011 & -1.553401 & 0.645767 \\ \mathrm{C} & -0.389660 & 0.184376 & 0.728299 \\ \mathrm{C} & -1.487593 & -0.486043 & -0.140826 \\ \mathrm{C} & -0.244561 & 2.389028 & -0.658335 \\ \mathrm{C} & 0.371460 & 1.319817 & 0.036714 \\ \mathrm{~N} & 1.741101 & 1.343425 & 0.160005 \\ \mathrm{C} & 2.542298 & 2.249965 & -0.494389 \\ \mathrm{C} & 1.960247 & 3.218845 & -1.303652 \\ \mathrm{C} & 0.561379 & 3.287348 & -1.371833 \\ \mathrm{O} & -0.978182 & 0.736040 & 1.897540 \\ \mathrm{H} & -0.267473 & 1.019915 & 2.450257 \\ \mathrm{C} & -1.727765 & 2.666386 & -0.640335 \\ \mathrm{H} & 0.549701 & -2.755547 & -1.834964 \\ \mathrm{H} & -1.492688 & -3.785228 & -0.782952 \\ \mathrm{H} & -0.022275 & -4.165890 & 0.166321 \\ \mathrm{H} & -1.892092 & -3.437436 & 1.681238 \\ \mathrm{H} & -0.658785 & -1.638597 & 2.906738 \\ \mathrm{H} & 0.480930 & -2.864110 & 2.306512 \\ \mathrm{H} & 1.345332 & -0.590885 & 1.847738 \\ \mathrm{H} & 1.876885 & -2.493011 & 0.301461 \\ \mathrm{H} & 1.813798 & -0.989543 & -0.641762 \\ \mathrm{H} & -1.653943 & -1.561336 & -2.043506 \\ \mathrm{H} & -0.302002 & -0.393518 & -1.985279 \\ \mathrm{H} & -3.083907 & -1.982062 & 0.008517 \\ \mathrm{H} & -2.783977 & -1.100490 & 1.528213 \\ \mathrm{H} & -2.240838 & 0.254737 & -0.484305 \\ \mathrm{H} & 3.638603 & 2.195774 & -0.384313 \\ \mathrm{H} & 2.587745 & 3.932452 & -1.863506 \\ \mathrm{H} & 0.099688 & 4.081268 & -1.982753 \\ \mathrm{H} & -1.924154 & 3.761501 & -0.692261 \\ \mathrm{H} & -2.232162 & 2.336379 & 0.293185 \\ \mathrm{H} & -2.221264 & 2.210156 & -1.528144\end{array}$


2-(2-Methoxy-adamantan-2-yl)-3-methyl-pyridine (6):

ground state

\begin{tabular}{rrrr}
$\mathrm{C}$ & 0.515171 & -2.411157 & -1.208819 \\
$\mathrm{C}$ & -0.430959 & -3.420983 & -0.539636 \\
$\mathrm{C}$ & -1.306998 & -2.686008 & 0.487997 \\
$\mathrm{C}$ & -0.418768 & -2.012251 & 1.545080 \\
$\mathrm{C}$ & 0.505444 & -0.978513 & 0.866697 \\
$\mathrm{C}$ & 1.391798 & -1.753766 & -0.134332 \\
$\mathrm{C}$ & -0.295398 & -1.314498 & -1.916124 \\
$\mathrm{C}$ & -2.126107 & -1.608138 & -0.234664 \\
$\mathrm{C}$ & -0.307601 & 0.164996 & 0.171649 \\
$\mathrm{C}$ & -1.175525 & -0.583102 & -0.886457 \\
$\mathrm{C}$ & 0.146286 & 2.251023 & -1.336695 \\
$\mathrm{C}$ & 0.591034 & 1.242683 & -0.445986 \\
$\mathrm{~N}$ & 1.910333 & 1.272554 & -0.057629 \\
$\mathrm{C}$ & 2.841722 & 2.125666 & -0.601534 \\
$\mathrm{C}$ & 2.449779 & 3.030947 & -1.580402 \\
$\mathrm{C}$ & 1.094889 & 3.091752 & -1.936151 \\
$\mathrm{O}$ & -1.206399 & 0.777899 & 1.107954 \\
$\mathrm{C}$ & -0.587076 & 1.553174 & 2.132100 \\
$\mathrm{C}$ & -1.302773 & 2.527040 & -1.655371 \\
$\mathrm{H}$ & 1.160210 & -2.942261 & -1.952420 \\
$\mathrm{H}$ & -1.068932 & -3.918701 & -1.307827 \\
$\mathrm{H}$ & 0.156106 & -4.226554 & -0.038143 \\
$\mathrm{H}$ & -1.994732 & -3.416771 & 0.981859 \\
$\mathrm{H}$ & -1.048117 & -1.536127 & 2.332592 \\
$\mathrm{H}$ & 0.188548 & -2.785717 & 2.072460 \\
$\mathrm{H}$ & 1.175138 & -0.563256 & 1.656613 \\
$\mathrm{H}$ & 1.985113 & -2.533470 & 0.400008 \\
$\mathrm{H}$ & 2.142557 & -1.096979 & -0.628426 \\
$\mathrm{H}$ & -0.930342 & -1.770399 & -2.712482 \\
$\mathrm{H}$ & 0.386432 & -0.596242 & -2.429220 \\
$\mathrm{H}$ & -2.771267 & -2.083754 & -1.011324 \\
$\mathrm{H}$ & -2.824751 & -1.110925 & 0.478421 \\
$\mathrm{H}$ & -1.831586 & 0.125392 & -1.436164 \\
$\mathrm{H}$ & 3.892186 & 2.078740 & -0.268509 \\
$\mathrm{H}$ & 3.187843 & 3.700788 & -2.052501 \\
$\mathrm{H}$ & 0.780079 & 3.835061 & -2.687802 \\
$\mathrm{H}$ & -1.392759 & 1.984895 & 2.772890 \\
$\mathrm{H}$ & 0.060372 & 0.922766 & 2.783532 \\
$\mathrm{H}$ & -0.005708 & 2.401792 & 1.702007 \\
\hline & -1.465499 & 3.614233 & -1.834500 \\
$\mathrm{H}$ & -1.604412 & 2.000951 & -2.589247
\end{tabular}




$\begin{array}{lrrr}\text { transition state } & & \\ & & & \\ \mathrm{C} & 1.285180 & -2.091530 & -0.693465 \\ \mathrm{C} & 1.269949 & -2.416998 & -2.195790 \\ \mathrm{C} & 0.760150 & -1.190897 & -2.971045 \\ \mathrm{C} & -0.657307 & -0.829617 & -2.498820 \\ \mathrm{C} & -0.633568 & -0.482299 & -0.996011 \\ \mathrm{C} & -0.138361 & -1.734896 & -0.240352 \\ \mathrm{C} & 2.208396 & -0.892355 & -0.424777 \\ \mathrm{C} & 1.699297 & -0.005228 & -2.705821 \\ \mathrm{C} & 0.275912 & 0.750530 & -0.710043 \\ \mathrm{C} & 1.696137 & 0.335043 & -1.201080 \\ \mathrm{C} & -0.129145 & 2.441138 & 1.164060 \\ \mathrm{C} & 0.285344 & 1.160971 & 0.758929 \\ \mathrm{~N} & 0.623812 & 0.248611 & 1.731324 \\ \mathrm{C} & 0.653735 & 0.578681 & 3.066025 \\ \mathrm{C} & 0.312622 & 1.867717 & 3.474363 \\ \mathrm{C} & -0.090574 & 2.805322 & 2.514005 \\ \mathrm{O} & -0.112493 & 1.888746 & -1.494145 \\ \mathrm{H} & -1.390881 & 2.437906 & -1.182080 \\ \mathrm{H} & 1.650626 & -2.982389 & -0.124363 \\ \mathrm{H} & 2.294112 & -2.695031 & -2.540335 \\ \mathrm{H} & 0.614024 & -3.297830 & -2.393897 \\ \mathrm{H} & 0.745043 & -1.422735 & -4.065069 \\ \mathrm{H} & -1.059442 & 0.018633 & -3.100392 \\ \mathrm{H} & -1.346318 & -1.687545 & -2.684504 \\ \mathrm{H} & -1.681727 & -0.283252 & -0.668862 \\ \mathrm{H} & -0.823697 & -2.594867 & -0.431010 \\ \mathrm{H} & -0.146999 & -1.582998 & 0.862900 \\ \mathrm{H} & 3.250259 & -1.139426 & -0.738884 \\ \mathrm{H} & 2.253111 & -0.677317 & 0.668582 \\ \mathrm{H} & 2.733259 & -0.260203 & -3.039992 \\ \mathrm{H} & 1.390736 & 0.874705 & -3.317316 \\ \mathrm{H} & 2.417741 & 1.175043 & -1.064120 \\ \mathrm{H} & -0.499243 & 3.177344 & 0.434280 \\ \mathrm{H} & 0.950871 & -0.178894 & 3.810733 \\ \mathrm{H} & 0.349862 & 2.142099 & 4.541827 \\ \mathrm{H} & -0.385328 & 3.822453 & 2.823875\end{array}$




$\begin{array}{lrrr}\text { transition state } & & \\ & & & \\ \mathrm{C} & -0.295898 & -2.710653 & -0.129327 \\ \mathrm{C} & -1.044380 & -3.376314 & -1.295424 \\ \mathrm{C} & -1.525183 & -2.289255 & -2.270646 \\ \mathrm{C} & -2.458367 & -1.310709 & -1.539616 \\ \mathrm{C} & -1.697083 & -0.628640 & -0.384287 \\ \mathrm{C} & -1.243823 & -1.738854 & 0.589003 \\ \mathrm{C} & 0.913360 & -1.924896 & -0.661116 \\ \mathrm{C} & -0.306918 & -1.522408 & -2.805438 \\ \mathrm{C} & -0.483973 & 0.196636 & -0.909669 \\ \mathrm{C} & 0.430258 & -0.835902 & -1.636955 \\ \mathrm{C} & 0.395862 & 2.330930 & 0.188266 \\ \mathrm{C} & 0.265600 & 0.931674 & 0.196735 \\ \mathrm{~N} & 0.760726 & 0.238766 & 1.277158 \\ \mathrm{C} & 1.449887 & 0.860431 & 2.292066 \\ \mathrm{C} & 1.647133 & 2.240511 & 2.258942 \\ \mathrm{C} & 1.107365 & 2.983457 & 1.200263 \\ \mathrm{O} & -0.884981 & 1.157326 & -1.898338 \\ \mathrm{C} & -1.721802 & 2.209564 & -1.423303 \\ \mathrm{H} & 0.051728 & -3.497150 & 0.585918 \\ \mathrm{H} & -0.375704 & -4.099296 & -1.819975 \\ \mathrm{H} & -1.913684 & -3.962045 & -0.912617 \\ \mathrm{H} & -2.072423 & -2.767899 & -3.120548 \\ \mathrm{H} & -2.862388 & -0.556545 & -2.254449 \\ \mathrm{H} & -3.345369 & -1.860394 & -1.144196 \\ \mathrm{H} & -2.413424 & 0.028676 & 0.163496 \\ \mathrm{H} & -2.130282 & -2.292965 & 0.979727 \\ \mathrm{H} & -0.732595 & -1.319963 & 1.485406 \\ \mathrm{H} & 1.618221 & -2.619261 & -1.177073 \\ \mathrm{H} & 1.481927 & -1.472569 & 0.184974 \\ \mathrm{H} & 0.380400 & -2.225888 & -3.333131 \\ \mathrm{H} & -0.626464 & -0.777905 & -3.571685 \\ \mathrm{H} & 1.319865 & -0.326924 & -2.078142 \\ \mathrm{H} & -0.063722 & 2.940700 & -0.604492 \\ \mathrm{H} & 1.847494 & 0.263005 & 3.129721 \\ \mathrm{H} & 2.212350 & 2.741939 & 3.062233 \\ \mathrm{H} & 1.235543 & 4.079037 & 1.171887 \\ \mathrm{H} & -1.217407 & 2.799271 & -0.622722 \\ \mathrm{H} & -2.701530 & 1.817710 & -1.065618 \\ \mathrm{H} & -1.925856 & 2.899755 & -2.276590\end{array}$


3-Pyridin-2-yl-adamantan-2-ol (3):

\section{transition state}

$\begin{array}{lrrr}\mathrm{C} & -2.054912 & -0.376971 & -0.789161 \\ \mathrm{C} & -2.302108 & -0.981376 & -2.187763 \\ \mathrm{C} & -1.228676 & -2.035547 & -2.497398 \\ \mathrm{C} & 0.153499 & -1.372202 & -2.428926 \\ \mathrm{C} & 0.369957 & -0.764262 & -1.027607 \\ \mathrm{C} & -0.677151 & 0.350266 & -0.703253 \\ \mathrm{C} & -2.150648 & -1.530603 & 0.233398 \\ \mathrm{C} & -1.296144 & -3.167816 & -1.459842 \\ \mathrm{C} & 0.308535 & -1.900715 & 0.009854 \\ \mathrm{C} & -1.068151 & -2.580161 & -0.057773 \\ \mathrm{~N} & -0.239722 & 0.852757 & 3.056086 \\ \mathrm{C} & -0.441641 & 0.262969 & 1.832777 \\ \mathrm{C} & -0.378243 & 1.011139 & 0.645234 \\ \mathrm{C} & 0.058727 & 2.344181 & 0.751048 \\ \mathrm{C} & 0.265205 & 2.936743 & 2.004272 \\ \mathrm{C} & 0.095445 & 2.178425 & 3.162599 \\ \mathrm{O} & -0.544249 & 1.339692 & -1.735863 \\ \mathrm{H} & -1.376328 & 2.488235 & -1.591799 \\ \mathrm{H} & -2.893273 & 0.331321 & -0.593666 \\ \mathrm{H} & -3.312913 & -1.452464 & -2.230698 \\ \mathrm{H} & -2.304054 & -0.189809 & -2.973017 \\ \mathrm{H} & -1.396123 & -2.456632 & -3.519999 \\ \mathrm{H} & 0.945067 & -2.128269 & -2.647286 \\ \mathrm{H} & 0.248085 & -0.595033 & -3.223195 \\ \mathrm{H} & 1.403193 & -0.344252 & -1.034832 \\ \mathrm{H} & -2.034825 & -1.169181 & 1.280144 \\ \mathrm{H} & -3.160207 & -2.003654 & 0.186938 \\ \mathrm{H} & -0.525653 & -3.943654 & -1.682456 \\ \mathrm{H} & -2.288295 & -3.676195 & -1.508828 \\ \mathrm{H} & 1.110149 & -2.650659 & -0.190720 \\ \mathrm{H} & 0.496003 & -1.508471 & 1.036852 \\ \mathrm{H} & -1.121287 & -3.399644 & 0.701501 \\ \mathrm{H} & -0.650071 & -0.817268 & 1.805204 \\ \mathrm{H} & 0.253456 & 2.965607 & -0.135606 \\ \mathrm{H} & 0.567515 & 3.995018 & 2.077836 \\ \mathrm{H} & 0.243576 & 2.631121 & 4.157496\end{array}$




\section{transition state}

$\begin{array}{rrrr}\text { C } & -0.666186 & -0.510701 & -0.936830 \\ \mathrm{C} & -0.548627 & -0.927372 & -2.418380 \\ \mathrm{C} & 0.638244 & -1.885737 & -2.598054 \\ \mathrm{C} & 1.919368 & -1.183652 & -2.128020 \\ \mathrm{C} & 1.771100 & -0.765075 & -0.650471 \\ \mathrm{C} & 0.602228 & 0.252074 & -0.443057 \\ \mathrm{C} & -0.908694 & -1.796299 & -0.115850 \\ \mathrm{C} & 0.418201 & -3.151137 & -1.753357 \\ \mathrm{C} & 1.557255 & -2.034386 & 0.194956 \\ \mathrm{C} & 0.282457 & -2.752265 & -0.275016 \\ \mathrm{~N} & 0.144548 & 0.252291 & 3.347272 \\ \mathrm{C} & 0.265507 & -0.172058 & 2.047154 \\ \mathrm{C} & 0.542333 & 0.733530 & 1.009204 \\ \mathrm{C} & 0.849513 & 2.055881 & 1.378342 \\ \mathrm{C} & 0.726052 & 2.479012 & 2.708723 \\ \mathrm{C} & 0.353118 & 1.563123 & 3.692449 \\ \mathrm{O} & 0.894730 & 1.377988 & -1.285463 \\ \mathrm{C} & -0.026920 & 2.462077 & -1.197742 \\ \mathrm{H} & -1.574594 & 0.130248 & -0.855576 \\ \mathrm{H} & -1.488129 & -1.427917 & -2.753562 \\ \mathrm{H} & -0.429053 & -0.036442 & -3.078145 \\ \mathrm{H} & 0.735586 & -2.169242 & -3.675641 \\ \mathrm{H} & 2.790788 & -1.870638 & -2.248154 \\ \mathrm{H} & 2.135754 & -0.301895 & -2.775727 \\ \mathrm{H} & 2.747286 & -0.307345 & -0.364909 \\ \mathrm{H} & -1.057529 & -1.577256 & 0.965619 \\ \mathrm{H} & -1.846108 & -2.298300 & -0.454372 \\ \mathrm{H} & 1.271560 & -3.857933 & -1.885015 \\ \mathrm{H} & -0.499118 & -3.687062 & -2.094573 \\ \mathrm{H} & 2.434122 & -2.717295 & 0.095016 \\ \mathrm{H} & 1.480368 & -1.779246 & 1.277902 \\ \mathrm{H} & 0.117137 & -3.669488 & 0.343444 \\ \mathrm{H} & 0.144937 & -1.245789 & 1.838004 \\ \mathrm{H} & 1.194977 & 2.800009 & 0.645486 \\ \mathrm{H} & 0.928980 & 3.528448 & 2.981335 \\ \mathrm{H} & 0.241008 & 1.880927 & 4.742742 \\ \mathrm{H} & 0.310527 & 3.260617 & -1.901031 \\ \mathrm{H} & -0.040837 & 2.897897 & -0.171595 \\ \mathrm{H} & -1.051037 & 2.149310 & -1.505356\end{array}$


2-(3-Methyl-pyridin-2-yl)-adamantan-2-ol (5) :

Transition state

$\begin{array}{rrrr}\mathrm{C} & 0.087250 & -2.276820 & -0.935989 \\ \mathrm{C} & -0.698431 & -3.333565 & -0.142368 \\ \mathrm{C} & -1.324138 & -2.671806 & 1.096258 \\ \mathrm{C} & -0.220144 & -2.062277 & 1.973236 \\ \mathrm{C} & 0.543790 & -0.983802 & 1.175628 \\ \mathrm{C} & 1.186636 & -1.685640 & -0.040180 \\ \mathrm{C} & -0.857470 & -1.148144 & -1.379975 \\ \mathrm{C} & -2.274011 & -1.553401 & 0.645767 \\ \mathrm{C} & -0.389660 & 0.184376 & 0.728299 \\ \mathrm{C} & -1.487593 & -0.486043 & -0.140826 \\ \mathrm{C} & -0.244561 & 2.389028 & -0.658335 \\ \mathrm{C} & 0.371460 & 1.319817 & 0.036714 \\ \mathrm{~N} & 1.741101 & 1.343425 & 0.160005 \\ \mathrm{C} & 2.542298 & 2.249965 & -0.494389 \\ \mathrm{C} & 1.960247 & 3.218845 & -1.303652 \\ \mathrm{C} & 0.561379 & 3.287348 & -1.371833 \\ \mathrm{O} & -0.978182 & 0.736040 & 1.897540 \\ \mathrm{H} & -0.267473 & 1.019915 & 2.450257 \\ \mathrm{C} & -1.727765 & 2.666386 & -0.640335 \\ \mathrm{H} & 0.549701 & -2.755547 & -1.834964 \\ \mathrm{H} & -1.492688 & -3.785228 & -0.782952 \\ \mathrm{H} & -0.022275 & -4.165890 & 0.166321 \\ \mathrm{H} & -1.892092 & -3.437436 & 1.681238 \\ \mathrm{H} & -0.658785 & -1.638597 & 2.906738 \\ \mathrm{H} & 0.480930 & -2.864110 & 2.306512 \\ \mathrm{H} & 1.345332 & -0.590885 & 1.847738 \\ \mathrm{H} & 1.876885 & -2.493011 & 0.301461 \\ \mathrm{H} & 1.813798 & -0.989543 & -0.641762 \\ \mathrm{H} & -1.653943 & -1.561336 & -2.043506 \\ \mathrm{H} & -0.302002 & -0.393518 & -1.985279 \\ \mathrm{H} & -3.083907 & -1.982062 & 0.008517 \\ \mathrm{H} & -2.783977 & -1.100490 & 1.528213 \\ \mathrm{H} & -2.240838 & 0.254737 & -0.484305 \\ \mathrm{H} & 3.638603 & 2.195774 & -0.384313 \\ \mathrm{H} & 2.587745 & 3.932452 & -1.863506 \\ \mathrm{H} & 0.099688 & 4.081268 & -1.982753 \\ \mathrm{H} & -1.924154 & 3.761501 & -0.692261 \\ \mathrm{H} & -2.232162 & 2.336379 & 0.293185 \\ \mathrm{H} & -2.221264 & 2.210156 & -1.528144\end{array}$


2-(2-Methoxy-adamantan-2-yl)-3-methyl-pyridine (6):

Transition state

\begin{tabular}{|c|c|c|c|}
\hline C & 0.045810 & -2.715005 & -0.199419 \\
\hline C & -0.501032 & -3.329491 & -1.487495 \\
\hline C & -0.664816 & -2.244422 & -2.551378 \\
\hline C & -1.628790 & -1.176182 & -2.035337 \\
\hline C & -1.075653 & -0.543343 & -0.756596 \\
\hline C & -0.929721 & -1.647077 & 0.293696 \\
\hline C & 1.400160 & -2.067555 & -0.487560 \\
\hline C & 0.696712 & -1.605629 & -2.823257 \\
\hline C & 0.284275 & 0.113415 & -1.032463 \\
\hline C & 1.241553 & -0.970948 & -1.542426 \\
\hline C & 1.027479 & 2.219888 & 0.188903 \\
\hline C & 0.835588 & 0.824981 & 0.181646 \\
\hline $\mathrm{N}$ & 1.112964 & 0.109912 & 1.317349 \\
\hline C & 1.624616 & 0.708528 & 2.446971 \\
\hline C & 1.875871 & 2.076405 & 2.454402 \\
\hline C & 1.560840 & 2.839798 & 1.318565 \\
\hline O & 0.101539 & 1.050654 & -2.075035 \\
\hline C & -0.517855 & 1.695872 & -1.773247 \\
\hline C & 0.659276 & 3.064838 & -0.992492 \\
\hline $\mathrm{H}$ & 0.164820 & -3.494573 & 0.567541 \\
\hline $\mathrm{H}$ & 0.173805 & -4.132028 & -1.851957 \\
\hline $\mathrm{H}$ & -1.470784 & -3.834676 & -1.295566 \\
\hline $\mathrm{H}$ & -1.062196 & -2.684967 & -3.477706 \\
\hline $\mathrm{H}$ & -1.796914 & -0.399823 & -2.810765 \\
\hline $\mathrm{H}$ & -2.630336 & -1.616818 & -1.848024 \\
\hline $\mathrm{H}$ & -1.762574 & 0.235023 & -0.392906 \\
\hline $\mathrm{H}$ & -0.581723 & -1.221183 & 1.258032 \\
\hline $\mathrm{H}$ & -1.917491 & -2.100362 & 0.519670 \\
\hline $\mathrm{H}$ & 2.129948 & -2.831395 & -0.828550 \\
\hline $\mathrm{H}$ & 1.835800 & -1.649777 & 0.444105 \\
\hline $\mathrm{H}$ & 0.614185 & -0.845673 & -3.628304 \\
\hline $\mathrm{H}$ & 1.408492 & -2.362457 & -3.214484 \\
\hline $\mathrm{H}$ & 2.215123 & -0.502040 & -1.748266 \\
\hline $\mathrm{H}$ & 1.831301 & 0.097620 & 3.338159 \\
\hline $\mathrm{H}$ & 2.317041 & 2.547490 & 3.345162 \\
\hline $\mathrm{H}$ & 1.732183 & 3.926318 & 1.313624 \\
\hline $\mathrm{H}$ & -0.691370 & 2.454746 & -2.564462 \\
\hline $\mathrm{H}$ & -1.483097 & 1.208084 & -1.524011 \\
\hline $\mathrm{H}$ & -0.126202 & 2.203387 & -0.867111 \\
\hline $\mathrm{H}$ & 0.880104 & 4.131515 & -0.779876 \\
\hline $\mathrm{H}$ & -0.423267 & 2.958110 & -1.212776 \\
\hline $\mathrm{H}$ & 1.240966 & 2.746941 & -1.882630 \\
\hline
\end{tabular}

